\title{
SISTEMA COMPUTARIZADO PARA LA GESTIÓN DEL AGUA EN SISTEMAS DE RIEGO POR GRAVEDAD EN MÉXICO
}

\author{
Martín D. Mundo Molina ${ }^{1}$ y Polioptro Martínez Austria²
}

\begin{abstract}
Resumen:
En México los distritos de riego enfrentan diversos problemas, tanto técnicos como de gestión administrativa. En la parte administrativa es preciso automatizar grandes volúmenes de información, de tal manera que ésta sea expedida. En la parte técnica es necesario, entre otros importantes y diversos problemas, mejorar la eficiencia de los distritos, maximizando la producción por unidad de agua utilizada. Una alternativa para apoyarlos en la solución de esta problemática es la incorporación y uso de diversas herramientas tecnológicas, software y hardware, que integradas permitan facilitar la gestión técnica y administrativa de dichos sistemas de riego. Con estos objetivos se creó el SICODE. El SICODE es un sistema de cómputo que integra de manera modular los siguientes programas: base de datos, sistema de información geográfica, simulación de balance de agua en el suelo (pronóstico del riego, apoyado en estaciones meteorológicas automatizadas), simulador biológico y sistema experto. El objetivo de este trabajo es presentar las principales características del SICODE, que actualmente está implementado en el distrito de riego: 010 Culiacán en el estado de Sinaloa, México.
\end{abstract}

Palabras clave: demandas de riego, pronóstico de riego, evapotranspiración del cultivo de referencia, rendimiento de riego, distritos de riego, sicode.

\section{INTRODUCCIÓN}

En México, actualmente prevalece el riego por gravedad y la cultura del riego tradicional, donde la decisión del momento del riego y la cantidad del mismo la toma el usuario con base en su criterio que, en pocos casos no es del todo inadecuado, sin embargo y en general, cuando es incorrecto el dispendio es grande (Mundo et al, 1995). Los bajos rendimientos en el uso del agua de riego, tanto a nivel parcelario como en los canales de distribución, provocan a su vez bajos rendimientos globales en la mayoría de los distritos de riego del país.

Existen muchas alternativas técnicas para mejorar el uso del agua en los sistemas de riego, una de ellas es la implementación de metodologías para determinar las fechas y cantidades de riego (cuándo y cuánto regar), ligadas a sistemas computarizados que facilitan el tratamiento ingente de datos meteorológicos, de suelo, cultivos, parcelas y usuarios.
Actualmente los sistemas computarizados (software y hardware) constituidos por bases de datos, sistemas de información geográfica, medición de variables meteorológicas en tiempo real con estaciones automatizadas, facilitan la puesta en práctica de estas técnicas en campo.

Los métodos de balance de agua permiten estimar los cambios de humedad del suelo de manera indirecta, considerando lo que se evapotranspira, llueve y se aporta con el agua de riego, que aplicado en el momento oportuno y en la cantidad necesaria permite no sólo ahorrar agua, sino además evita el decremento en el rendimiento de los cultivos.

Considerando estos aspectos se desarrolló el SICODE, como una herramienta de apoyo a los agricultores, para mejorar estos dos insumos en el quehacer diario de los distritos de riego, por un lado la gestión administrativa, que a través de módu-

Instituto Mexicano de Tecnología del Agua. Paseo Cuaunháhuac 8532, Jiutepec, Morelos, México

1. Email: mmundo@iru.etsia.upm.es. Tel.: 913365675 FAX: 913365845

2. Tel. (73) 1936 63. Email: polioptr@tlaloc.imta.mx

Artículo recibido el 30 de agosto de 2000, recibido en forma revisada el 4 de octubre de 2001 y el 28 de diciembre de 2001 y aceptado para su publicación el 15 de enero de 2002. Pueden ser remitidas discusiones sobre el artículo hasta seis meses después de la publicación del mismo siguiendo lo indicado en las "Instrucciones para autores". En el caso de ser aceptadas, éstas serán publicadas conjuntamente con la respuesta de los autores. 
los integrados proporcionan al agricultor recomendaciones sobre aspectos administrativos, por ejemplo: pagos, cobros, padrón de usuarios, permisos de siembra, contratos, tarifas de riego, asignación de volúmenes de agua, riegos solicitados, riegos en proceso, riegos aplicados; y por otro, la parte técnica, que pronostica el momento y la cantidad adecuada de riego.

Desde su concepción hasta su puesta en práctica en campo, el SICODE ha pasado por varias etapas. La etapa de investigación se inició en 1994 y concluyó con el desarrollo del modelo de balance de agua, el sistema experto y las bases de datos relacionales, acoplados a un sistema de información geográfica. Durante 1995 se validó en gabinete y en campo, iniciando las primeras pruebas en el distrito de riego 05, Delicias, en el estado de Chihuahua, en el norte del país. Así, SICODE se convirtió en pionero en la instalación y uso de estaciones meteorológicas automatizadas para fines de riego en México. En 1996 inició su divulgación y promoción mediante videos y cursos de capacitación; en ese mismo año se capacitaron a jefes de operación y gerentes de los principales distritos de riego del país. En 1997 inició su transferencia, primero a pequeñas superficies de riego, para luego pasar a áreas cada vez más grandes. Promocionado por los propios usuarios, durante 1997 se transfirió a los distritos de riego 038 en Navojoa, Sonora y 010 en Culiacán en el estado de Sinaloa (Mundo et al, 1997 a, 1998).

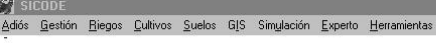

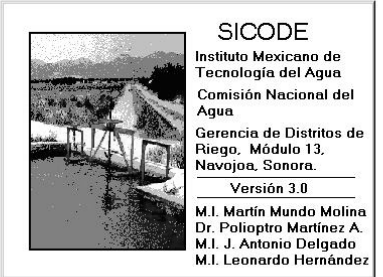

Figura.1 Menú principal del SICODE

\section{COMPONENTES DEL SICODE}

El SICODE es una herramienta de cómputo diseñado para distritos de riego, enfocado principalmente a dos aspectos: 1 . Gestión administrativa y 2. Pronóstico de riego.

El SICODE integra de manera modular los siguientes subsistemas (fig. 1):

- Base de datos

- Sistema de información geográfica

- Programación y pronóstico de riego

- Simulador biológico (IBSNAT, 1998) 3 , y

- Sistema experto

Tabla 1. Gestión administrativa

\begin{tabular}{|c|l|}
\hline SUBMENU & \multicolumn{1}{c|}{ SERVICIO QUE PRESTA } \\
\hline Padrón de usuarios & $\begin{array}{l}\text { Ubicación de usuarios dentro del distrito de riego. Proporciona además, para } \\
\text { cada usuario: número de cuenta, subcuenta, superficie física y con derecho a } \\
\text { riego, puntos entrega del agua de cada parcela, tipo de sistema de riego explo- } \\
\text { tado. } \\
\text { Estima la posible distribución del volumen de agua neto disponible para un ci- } \\
\text { clo agrícola determinado, para cada sección de riego dentro del distrito. Infor- } \\
\text { ma al usuario sobre el volumen de agua utilizado y el disponible para cada sec- } \\
\text { ción de riego. Realiza la estimación del volumen de agua disponible por hectá- } \\
\text { rea para todo el distrito de riego. } \\
\text { Permite imprimir el contrato de riego del usuario, en el cual se registra el volu- } \\
\text { men de agua que éste usará para un cultivo en determinado ciclo agrícola, rie- } \\
\text { gos disponibles, caudal a usar, duración y pagos por concepto de riego. } \\
\text { Elabora el permiso de siembra de cada usuario. Aquí se registra: el nombre del } \\
\text { agricultor, superficie de cultivo, superficie de siembra, tipo de cultivo, variedad, } \\
\text { ciclo agrícola, número de cuenta y nombre de quien autoriza dicha siembra. } \\
\text { Lleva un control de pagos y tarifas de riego, en dos modalidades: por riego o } \\
\text { por volumen. } \\
\text { Informa sobre el balance entre pagos y débitos por concepto de riego, para ca- } \\
\text { da usuario del distrito. } \\
\text { Proporciona reportes de deudores por concepto de riego. }\end{array}$ \\
Tarifas & Pagos
\end{tabular}

3 El simulador biológico por su extensión sólo se enunciará en este artículo 
A continuación se presenta una descripción sucinta de la base de datos del SICODE, los servicios que brinda a los agricultores y personal técnico de las asociaciones de usuarios de los distritos de riego.

\section{EI SICODE COMO GESTOR ADMINISTRATIVO}

Lleva un control del padrón de usuarios, asignación de volúmenes de agua para un ciclo agríco- la, contratos, permisos de siembra, tarifas, pagos y reportes de deudores (tabla 1).

El menú Gestión administrativa se muestra el submenú padrón de usuarios (fig. 2).

\section{Riegos y recomendaciones técnicas de riego}

Lleva un control del número de riegos solicitados, en proceso y aplicados; además proporciona al usuario recomendaciones técnicas de riego (tabla 2).
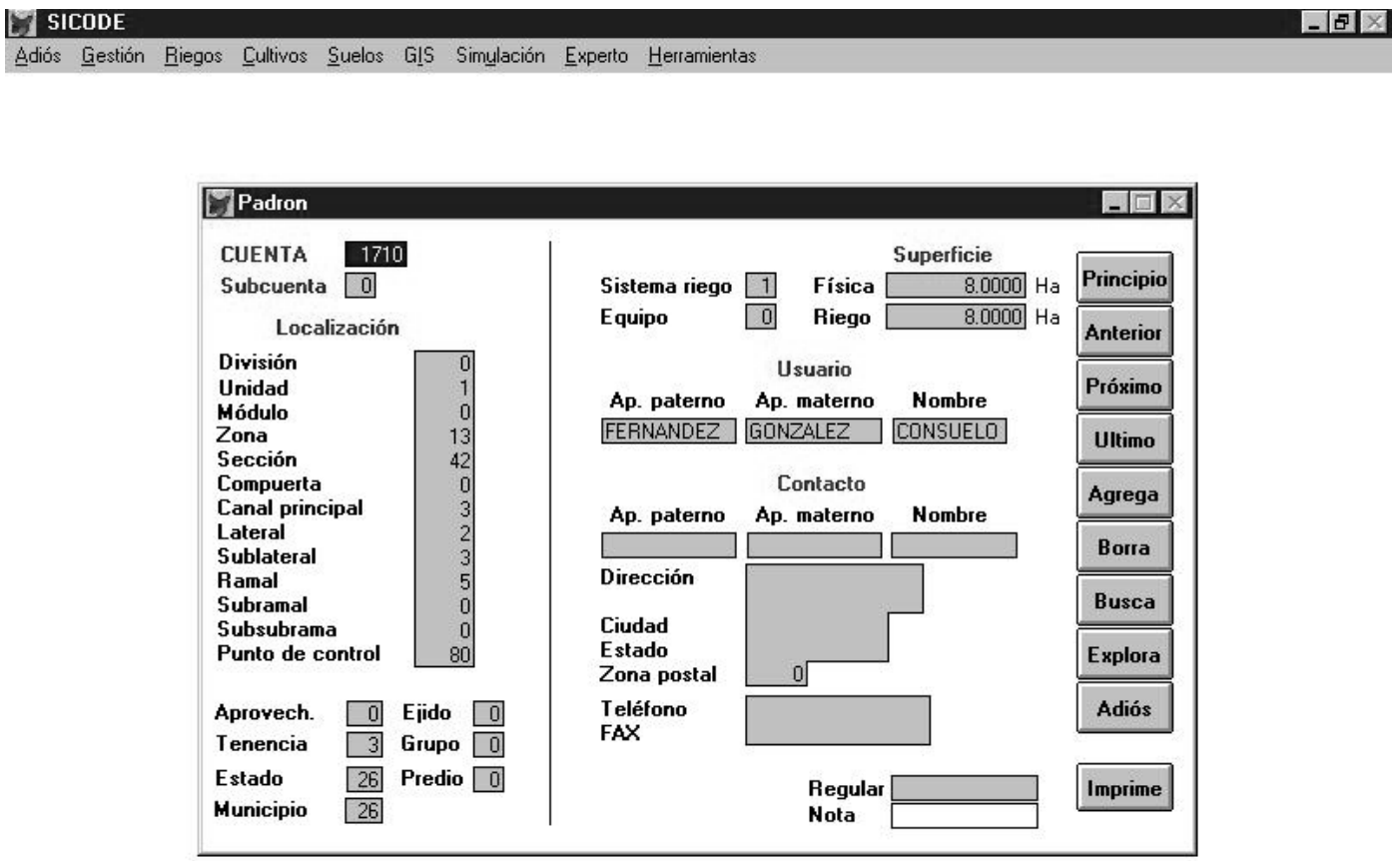

\begin{tabular}{|c|c|}
\hline \multirow{2}{*}{\multicolumn{2}{|c|}{$\begin{array}{l}\text { Padron Reg: 1/384 } \\
\text { Figura. } 2 \text { Padrón de usuarios }\end{array}$}} \\
\hline & \\
\hline SUBMENU & SERVICIO QUE PRESTA \\
\hline Riegos solicitados & $\begin{array}{l}\text { Informa el nombre y número de cuenta de la persona que solicita el riego, fe- } \\
\text { cha y ciclo agrícola de la solicitud, caudal a distribuir, caudal promedio por día y } \\
\text { por riego, duración estimada del riego. }\end{array}$ \\
\hline Riegos en proceso & $\begin{array}{l}\text { Proporciona el nombre del usuario, número de cuenta y la parcela correspon- } \\
\text { diente bajo riego, cultivo que se riega y caudal usado. }\end{array}$ \\
\hline Riegos aplicados & $\begin{array}{l}\text { Informa el nombre y código del usuario que aplicó el riego, fecha y ciclo agríco- } \\
\text { la de la aplicación, superficie sembrada, superficie bajo riego, caudal servido, } \\
\text { duración del riego, fecha y hora de inicio, fecha y hora de conclusión y volumen } \\
\text { usado. }\end{array}$ \\
\hline $\begin{array}{l}\text { Recomendaciones } \\
\text { técnicas de riego }\end{array}$ & $\begin{array}{l}\text { En función de las características físicas de la parcela y del cultivo explotado, su- } \\
\text { giere al usuario: ancho de surcos, longitud máxima de surcos, caudal recomen- } \\
\text { dado, volumen y duración del riego. Informa también sobre el número e inter- } \\
\text { valos de riego recomendables para dicho cultivo. }\end{array}$ \\
\hline
\end{tabular}




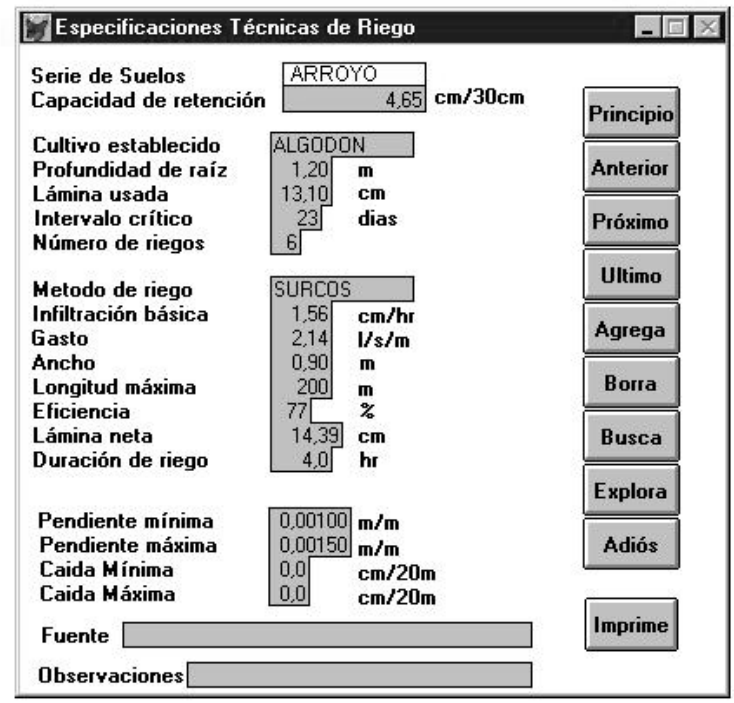

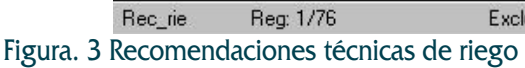

Tabla 3. Información sobre cultivos

\begin{tabular}{|c|l|}
\hline SUBMENU & \multicolumn{1}{|c|}{ SERVICIO QUE PRESTA } \\
\hline Perennes & $\begin{array}{l}\text { Proporciona al usuario información sobre cultivos perennes, los que se cultivan } \\
\text { en cada región particular y las diversas variedades de los mismos. Muestra in- } \\
\text { formación sobre coeficientes de cultivos de cada ciclo vegetativo, descenso to- } \\
\text { lerable de humedad y umbral recomendable para tolerancia a la salinidad. }\end{array}$ \\
\hline Anuales & La información es idéntica a la que se describe en cultivos perennes. \\
\hline Información agronómica autorizados & $\begin{array}{l}\text { Informa al usuario sobre los cultivos autorizados para la siembra en cada ciclo } \\
\text { agrícola, así como la variedad de los mismos. }\end{array}$ \\
\hline $\begin{array}{l}\text { Este submenú proporciona información de cada cultivo contenido en la base de } \\
\text { datos, por ejemplo: variedades de cultivos que se siembran a nivel regional, } \\
\text { nes sobre fertilización, tipos de plagas y su adecuado manejo, control de enfer- } \\
\text { medades, control de malezas, días de cosecha, tipos de herbicidas y dosis re- } \\
\text { comendables. }\end{array}$ \\
\hline
\end{tabular}

En la figura 3 se muestra un ejemplo del submenú recomendaciones técnicas de riego.

\section{Información agronómica}

Este submenú del SICODE tiene los principales cultivos perennes y anuales del distrito de riego y proporciona información agronómica de cada uno de los cultivos de la región (tabla 3 ).

En la figura 4 se muestra el submenú información agronómica.

\section{Información sobre suelos}

Proporciona información sobre algunas propiedades morfológicas, físicas y químicas de los diferentes tipos de suelo del distrito de riego (tabla 4).

\section{EL SICODE COMO SIMULADOR}

\section{Simulación de balance de agua en el suelo}

El simulador de balance de agua del SICODE 


\begin{tabular}{|c|c|c|c|c|}
\hline \multicolumn{4}{|l|}{ BINFORMACION AGRONOMICA } & $-\square \underline{\square}$ \\
\hline \multicolumn{2}{|l|}{ Cultivo } & \multicolumn{2}{|l|}{ Fertilizacion } & \\
\hline \multirow{2}{*}{\multicolumn{2}{|c|}{ CEBOLLA }} & \multirow{2}{*}{$\begin{array}{l}\text { 180-80-00 } \\
\text { Dividirlo en } 4 \text { partes: } \\
\text { 1. Al camelloneo aplicar todo el fósforo y } \\
\text { un cuarto del nitrógeno. }\end{array}$} & & Principio \\
\hline & & & th & Anterior \\
\hline \multicolumn{2}{|l|}{ Variedades } & \multicolumn{2}{|l|}{ Plagas, insecticidas $y$ dosis } & \\
\hline White Grano & & \multirow{2}{*}{$\begin{array}{l}\text { TRIPS. Paratión Metílico CE } 631 \mathrm{lt} / \text { ha o } \\
\text { Gusatión } \mathrm{M} 202 \mathrm{lt} / \mathrm{ha} \text {. }\end{array}$} & 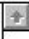 & Próximo \\
\hline $\begin{array}{l}\text { Híbrido Early Supreme } \\
\text { Hibrido White Tampico }\end{array}$ & & & \pm & Ultimo \\
\hline \multicolumn{2}{|l|}{ Epoca de siembra } & \multicolumn{2}{|l|}{ Enfermedades y control } & Agrega \\
\hline \multirow{2}{*}{$\begin{array}{|lr|}\text { INVIERNO } & \\
\text { Almácigo: } & 10-30 \mathrm{sep} \\
\text { Trasplante: } & 6 \cdot 26 \mathrm{nov} \\
\text { Directa: } & 10-31 \text { oct } \\
\end{array}$} & & \multirow{2}{*}{$\begin{array}{l}\text { RAIZ ROSA. Rotación de cultivos. } \\
\text { TIZON DEL FOLLAJE. Mancozeb } 3 \\
\text { It/ha, Captan PH } 503 \mathrm{~kg} / \mathrm{ha} \text {. Clorotalonil } \\
\mathrm{PH} 752 \mathrm{~kg} / \mathrm{ha} \text {. }\end{array}$} & $A$ & Borra \\
\hline & & & +4 & Busca \\
\hline \multicolumn{2}{|l|}{ Densidad } & \multicolumn{2}{|l|}{ Maleza, herbicidas y dosis } & \\
\hline 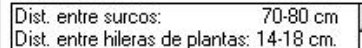 & & $\begin{array}{l}\text { Mantener limpio el cultivo por lo menos } \\
40 \text { días. }\end{array}$ & $t$ & Explora \\
\hline $\begin{array}{lr}\text { Dist entre plantas: } & 8 \mathrm{~cm} \\
\text { Cantidad de semilla: } & 2.5-4 \mathrm{~kg} / \mathrm{ha}\end{array}$ & L & $\begin{array}{l}\text { Los herbicidas para cebolla de trasplante } \\
\text { son: Dactal } W-75 \text { de } 10 \mathrm{a} 12 \mathrm{~kg} / \mathrm{ha} \text { y }\end{array}$ & If & Adiós \\
\hline \multicolumn{2}{|l|}{ Calendario de riego } & \multicolumn{2}{|l|}{ Dias a cosecha } & \\
\hline $\begin{array}{l}\text { - Riego de siembra o trasplante. } \\
\text { - Sobreriego de } 15 \text { a } 20 \text { días después del } \\
\text { primero. }\end{array}$ & & $\begin{array}{l}\text { INVIERND: Cuando el } 90 \% \text { de las } \\
\text { plantas doblen el follaje por su base en } \\
\text { invierno (210 a } 335 \text { días). }\end{array}$ & 1 & Imprime \\
\hline - Después, regar cada 20 a 25 días hasta & & PRIMAVERA: Casi no doblan el follaje & ta & \\
\hline
\end{tabular}

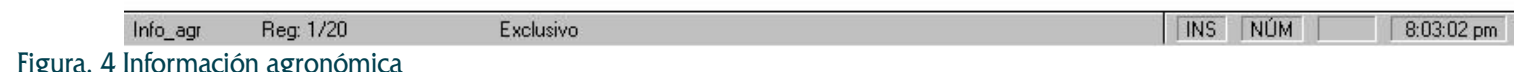

Tabla 4. Suelos

\begin{tabular}{|c|l|}
\hline SUBMENU & \multicolumn{1}{c|}{ SERVICIO QUE PRESTA } \\
\hline Morfología del suelo & $\begin{array}{l}\text { Informa al usuario sobre las características morfológicas de cada serie de suelo } \\
\text { del distrito, como: clasificación y profundidad de cada horizonte, color de cada } \\
\text { capa de suelo, textura, estructura, consistencia. }\end{array}$ \\
\hline Características físicas & $\begin{array}{l}\text { Informa al usuario sobre las características físicas de cada serie de suelo del dis- } \\
\text { trito como: profundidad de cada horizonte, pendiente, densidad aparente, po- } \\
\text { rosidad, textura (porcentaje de arena, limo y arcilla), capacidad de campo, pun- } \\
\text { to de marchitamiento permanente. }\end{array}$ \\
\hline Características químicas & $\begin{array}{l}\text { Informa al usuario sobre las características químicas de cada serie de suelo co- } \\
\text { mo: Ph, cantidad de materia orgánica, carbón orgánico, nitrógeno, carbonatos, } \\
\text { capacidad de intercambio catiónico, calcio, magnesio, potasio y conductividad } \\
\text { eléctrica. }\end{array}$ \\
\hline
\end{tabular}

toma datos meteorológicos diariamente de una estación automatizada. La estación mide cada cierto período de tiempo las siguientes variables: temperatura, humedad relativa, velocidad del viento, dirección del viento, radiación solar y precipitación; éstas son transmitidas vía radio o teléfono al SICODE. El intervalo de tiempo de las mediciones se define según el mejor criterio para almacenar la mayor cantidad de datos posible en la memoria del CR10 de la estación meteorológica automatizada. Los datos obtenidos se promedian cada 24 horas (temperatura, humedad relativa, velocidad del viento, radiación solar) o se suman en dicho período (precipitación). Con esta información se realiza un balance diario del contenido de humedad en el suelo, para cada cultivo establecido en el distrito (Mundo et al, 1997 b).

\section{El modelo}

Se han desarrollado una considerable cantidad de modelos que simulan el balance de humedad en el suelo y más recientemente el pronóstico del riego. Un problema común de todos son los datos del modelo, ya que es necesario introducir las variables más representativas para simular la dinámica del sistema agua-planta-suelo-atmósfera. Tal información comprende algunas características fí- 


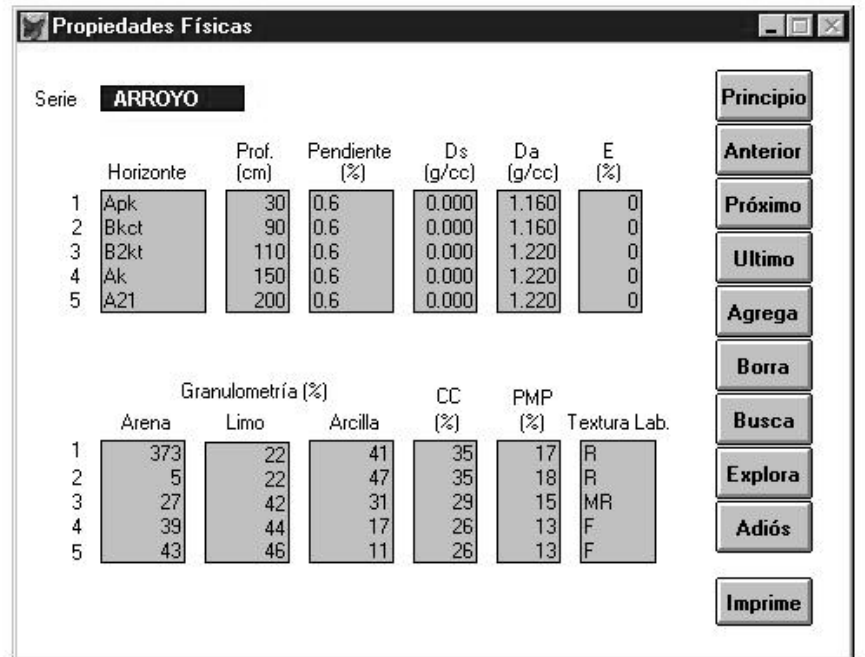

\section{Fisicas Reg: $1 / 3 \quad$ Exclusivo \\ Figura. 5 Propiedades físicas del suelo \\ sicas del suelo, datos del cultivo e información ac- tualizada del estado del tiempo meteorológico.}

Una vez definidos los datos correspondientes al suelo y cultivos, el pronóstico de riego, depende fundamentalmente de los datos meteorológicos. De la adecuada medición, precisión y exactitud de las variables meteorológicas depende a su vez la mejor aplicación de los modelos de simulación; para esta tarea el SICODE se apoya en estaciones meteorológicas automatizadas, las cuales envían datos al modelo, a intervalos de tiempo regulares.

Una forma de expresar el balance de agua en el suelo es:

$$
\mathrm{Bh}=\mathrm{Ss}(\uparrow)-\operatorname{Es}(\downarrow)
$$

De manera simplificada, se considera únicamente como salidas del sistema la evapotranspiración de los cultivos:

$$
\text { Ss }(\uparrow)=E t o
$$

Las entradas al sistema son la precipitación efectiva, la contribución de agua subterráneas y el agua almacenada antes del riego:

$$
\operatorname{Es}(\downarrow)=(\mathrm{Pe}+\mathrm{As}+\mathrm{Aa})
$$

Pero dado que para zonas áridas y balances diarios de agua en el suelo la ecuación (3) se puede sustituir por la precipitación total (Dastane, 1974), el balance de agua en el suelo se puede escribir de forma simplificada como:

$$
\mathrm{Bh}=\operatorname{Eto}(\uparrow)-\operatorname{Pt}(\downarrow)
$$

La evapotranspiración del cultivo de referencia, Eto, de la ecuación (4), se estima con la ecuación combinada de Penman-Monteith (Allen et al, 1998), con datos obtenidos diariamente de la estación meteorológica automatizada:

$$
\text { Eto }=\frac{0.408 \Delta(R n-G)+\gamma \frac{900}{T+273} U_{2}\left(e_{a}-e_{d}\right)}{\Delta+\gamma\left(1+0.34 U_{2}\right)}
$$

Para realizar el balance diario de humedad en el suelo (ec. 4), es necesario calcular la evolución en el tiempo de la humedad disponible para el cultivo, la humedad mínima tolerable por el mismo y la humedad residual del suelo. La humedad residual en el suelo en porcentaje de masa $\left(\theta_{\mathrm{i}}\right)$ en un instante de tiempo determinado, se puede estimar restando a dicha humedad residual la humedad del suelo en un tiempo posterior $\left(\theta_{i+1}\right)$, con la siguiente ecuación:

$$
\theta_{\mathrm{i}}=\theta_{\mathrm{i}}-\theta_{\mathrm{i}+1}
$$


Después de un riego y cuando el suelo alcanza su capacidad de campo (i=1), se cumple (ver fig. 6):

$$
\theta_{\mathrm{i}}=\mathrm{cc}
$$

Así, cuando el suelo pierde humedad por efecto de la radiación solar, el perfil húmedo empieza a descender. Para no afectar al cultivo, la humedad residual $\left(\theta_{i}\right)$ no debe descender por debajo de la humedad mínima tolerable por éste $\left(\theta_{t}\right)$, por lo tanto se debe verificar que:

$$
\theta_{\mathrm{t}} \geq \theta_{\mathrm{i}}
$$

donde se estima de la siguiente manera:

$$
\theta_{t}=c c-\left[\theta_{s}(D \text { th })\right]
$$

y la humedad disponible para el cultivo en porcentaje de masa $(\theta \mathrm{s})$ es $^{4}$ (ver fig.6):

$$
\theta_{\mathrm{s}}=\frac{\theta_{\mathrm{cc}}-\theta_{\mathrm{pmp}}}{100}
$$

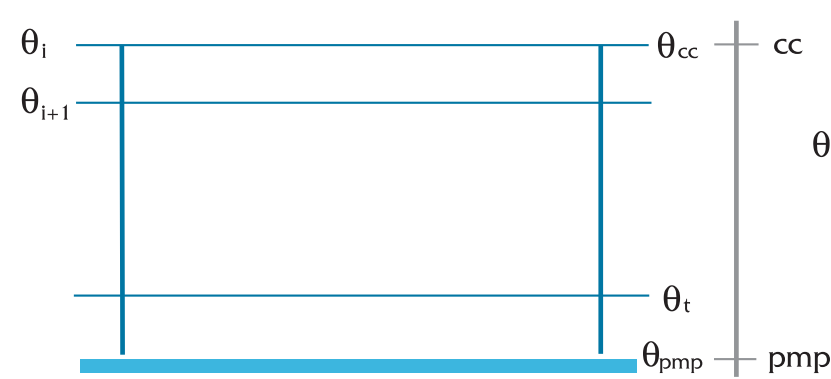

Figura 6. Perfil esquemático de la humedad del suelo

A continuación se presenta un ejemplo de balance de agua en el suelo. El SICODE ejecuta este procedimiento desde el menú simulación (ver fig.1). La simulación presentada en la tabla 5 fue realizada con datos de campo del módulo IV-1 del distrito de riego 010 Culiacán Sinaloa, para un suelo arcilloso, cuyas propiedades físicas son las siguientes: $\mathrm{cc}=42 \%, \mathrm{pmp}=27 \%, \mathrm{da}=1.02 \mathrm{gr} / \mathrm{cm}^{3}$. El cultivo considerado fue maíz, que alcanza una profundidad radical (Pr) de 1m; el descenso tolerable de humedad (Dth) para este cultivo se consideró de $70 \%$. La fecha de riego se calculó usando las ecuaciones (4), (5), (6), (9) y (10).

Posteriormente el programa calcula el volumen de agua que demanda el cultivo para cada par- cela simulada; si el usuario lo requiere, el SICODE puede pronosticar la fecha del siguiente riego.

\section{Pronóstico de riego}

El manejo de información meteorológica actual, en periodos cortos de tiempo, minimizando los factores aleatorios, se define como manejo de información meteorológica en tiempo real. Con esta información se realizan pronósticos de riego, cuyo objetivo es la estimación de la fecha futura de riego y los volúmenes de agua a aplicar.

Pronóstico de riego. El SICODE realiza el pronóstico de dos formas: 1.A través de un promedio móvil en la serie de Eto de la base de datos meteorológica, 2. Si las condiciones del clima en los últimos siete a diez días se mantienen sin cambios, se utilizan los últimos datos proyectándolos a futuro, para el mismo lapso de tiempo. Conocido Eto se pronostica a su vez la fecha de riego, de acuerdo al diagrama de flujo de la figura 7 .

$\theta_{\mathrm{s}}$

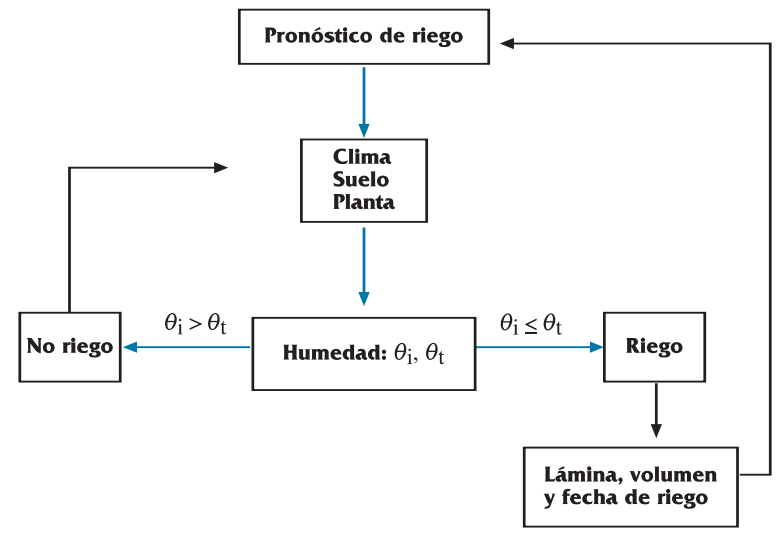

Figura 7. Diagrama de flujo para el pronóstico de la fecha de riego

\section{EL SISTEMA DE INFORMACIÓN GEOGRÁFICA}

Los sistemas de información geográfica (GIS, por sus siglas en inglés), son herramientas de cómputo que permiten al usuario manejar de manera rápida y expedita, gran cantidad de datos numéricos e información general de manera textual o gráfica. El GIS del SICODE está acoplado al simulador de balance de agua en el suelo, que una vez ejecutado presenta los resultados de manera gráfica, relacionando la información del GIS (mapa del distrito de riego digitalizado) con la base de datos numérica del simulador de balance de agua, presentando como resultado el mapa del distrito de riego. Así, el 
Tabla 5. Balance de agua en el suelo y cálculo de la fecha de riego

\begin{tabular}{|c|c|c|c|c|c|c|}
\hline Eto $(\mathrm{mm})$ & Pt (mm) & $\mathrm{Bh}=\mathrm{Eto}-\mathrm{Pt}(\mathrm{mm})$ & $\theta_{i+1}(\%)$ & $\theta_{1}(\%)$ & RIEGO & DIA JULIANO \\
\hline & & & & 42 (qcc) & & 1 \\
\hline 4.8 & 0 & 4.8 & 0.4706 & 41.5294 & NO & 2 \\
\hline 5.6 & 0 & 5.6 & 0.5490 & 40.9803 & NO & 3 \\
\hline 7.29 & 0 & 7.29 & 0.7147 & 40.2656 & NO & 4 \\
\hline 8 & 0 & 8 & 0.7843 & 39.4812 & NO & 5 \\
\hline 8.8 & 0 & 8.8 & 0.8627 & 38.6185 & NO & 6 \\
\hline 10.4 & 0 & 10.4 & 1.04 & 37.7585 & NO & 7 \\
\hline 11.2 & 0 & 11.2 & 1.12 & 36.6385 & $\mathrm{NO}$ & 8 \\
\hline 11.2 & 0 & 11.2 & 1.12 & 35.5185 & NO & 9 \\
\hline 11.2 & 0 & 11.2 & 1.12 & 34.3985 & $\mathrm{NO}$ & 10 \\
\hline 11.2 & 0 & 11.2 & 1.12 & 33.27 & NO & 11 \\
\hline 11.2 & 0 & 11.2 & 1.12 & 32.15 & NO & 12 \\
\hline 11.2 & 0 & 11.2 & 1.12 & 31.035 & Sî & 13 \\
\hline 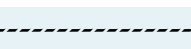 & 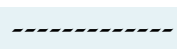 & $\mid$ & -..-.-.-.- & 42 & $\mathrm{NO}$ & 14 \\
\hline 4.8 & 0 & 4.8 & .4706 & 41.5294 & NO & 15 \\
\hline 5.6 & 0 & 5.6 & .5490 & 40.9803 & NO & 16 \\
\hline 7.2 & 0 & 7.2 & .7058 & 40.2744 & NO & 17 \\
\hline 4.704 & 0 & 4.704 & .4612 & 39.81 & NO & 18 \\
\hline 9.6 & 0 & 9.6 & .9411 & 38.86 & $\mathrm{NO}$ & 19 \\
\hline 12 & 0 & 12 & 1.2 & 37.66 & NO & 20 \\
\hline 8 & 0 & 8 & .7843 & 36.8756 & NO & 21 \\
\hline 8.8 & 50 & -32.4 & -3.1764 & 40.05 & NO & 22 \\
\hline 4.8 & 120 & -110.4 & -10.82 & 50.8 & NO & 23 \\
\hline$\cdots$ &  & 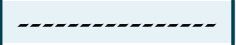 & --_------ & 42 & NO & 24 \\
\hline 7.92 & 0 & 7.92 & 0.7764 & 41.2235 & NO & 25 \\
\hline 9.6 & 0 & 9.6 & 0.9412 & 40.2823 & NO & 26 \\
\hline 8.2 & 0 & 8.2 & 0.8040 & 39.4783 & NO & 27 \\
\hline 8 & 0 & 8 & 0.7843 & 38.6940 & NO & 28 \\
\hline 7.3 & 0 & 7.3 & 0.7157 & 37.9783 & NO & 29 \\
\hline 8.5 & 0 & 8.5 & 0.8333 & 37.1450 & NO & 30 \\
\hline 9 & 0 & 9 & 0.8823 & 36.2627 & NO & 31 \\
\hline 9.5 & 0 & 9.5 & 0.9313 & 35.3314 & NO & 32 \\
\hline 8.9 & 0 & 8.9 & 0.8725 & 34.4589 & NO & 33 \\
\hline 8.5 & 0 & 8.5 & 0.8333 & 33.6256 & $\mathrm{NO}$ & 34 \\
\hline 9 & 0 & 9 & 0.8823 & 32.7433 & NO & 35 \\
\hline 9.8 & 0 & 9.8 & 0.9608 & 31.7825 & NO & 36 \\
\hline 9.9 & 0 & 9.9 & 0.9706 & 30.8119 & SI & 37 \\
\hline
\end{tabular}

GIS muestra todas las parcelas del distrito de riego (fig. 8), en donde el usuario puede observar (con base en un código de colores) los resultados del pronóstico de riego (Mundo et al., 1997 b), por ejemplo: cultivos que requieren riego inmediatamente (rojo), los que pueden esperar (azul) y los que no necesitan riego (verde). Además el GIS puede mostrar prácticamente la información deseada por el usuario (basta incorporarlo a la base de datos). El GIS usado por SICODE es el AU2WIN V. 3.0 (Dicartu, 1995).

\section{EL SISTEMA EXPERTO}

En períodos punta o de máxima demanda, los canales de distribución en los distritos de riego, no son capaces de conducir el caudal demandado por los cultivos, debido a la expansión de la superficie que éstos han tenido a lo largo del tiempo. Este problema también se presenta cuando ocurre una lluvia más o menos uniforme en dichos distritos. Días después de la lluvia, muchos cultivos demandan agua casi al mismo tiempo y los canales no tienen capacidad para conducir el caudal total requerido. 

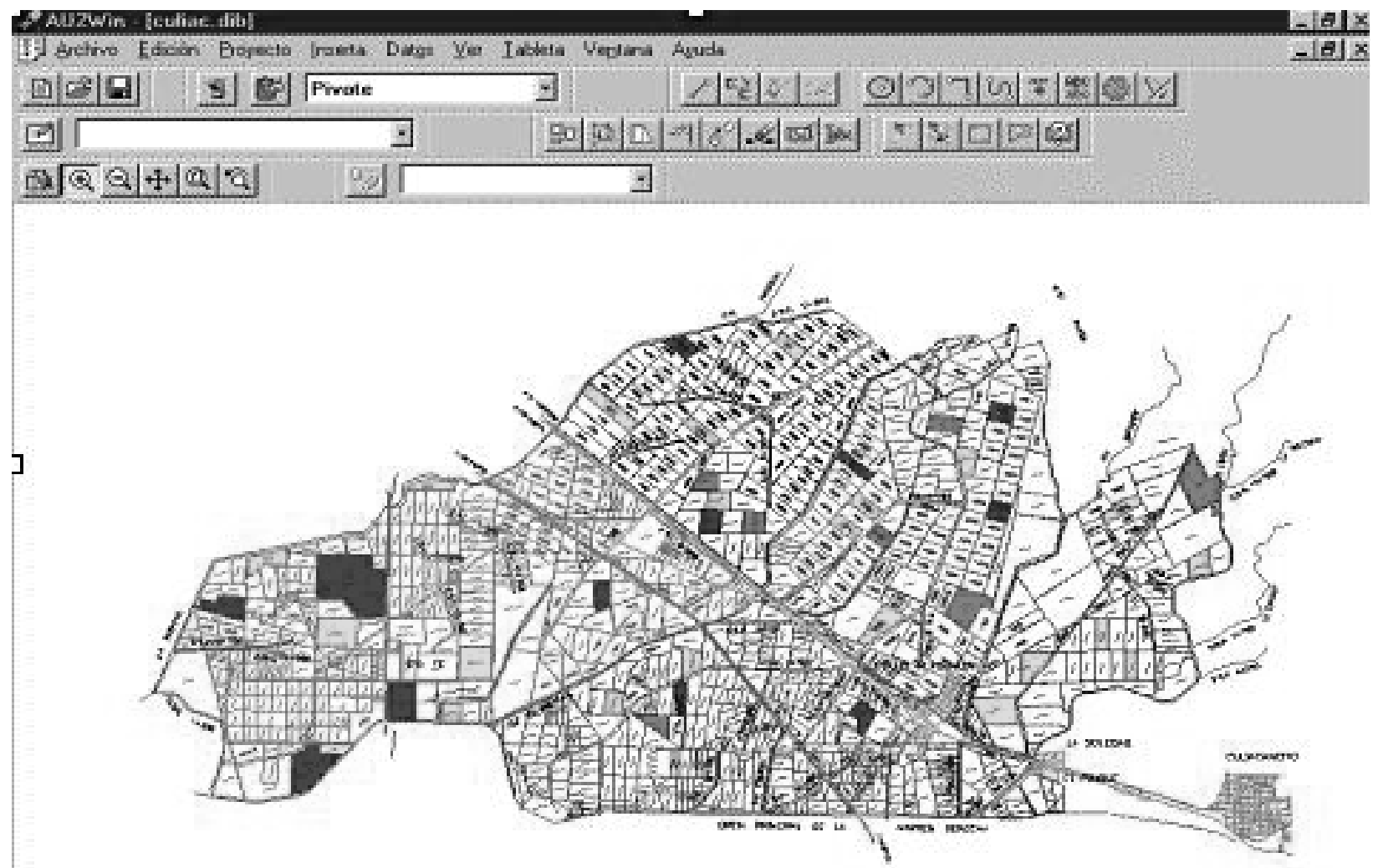

Lith

Figura 8. Resultado gráfico de la simulación (pronóstico de riego).

Ante este problema el administrador de la red de canales tiene que tomar, con base en su experiencia, ciertos criterios para repartir de la mejor forma el agua de riego a la mayoría de las parcelas demandantes, según el caudal disponible y la capacidad de conducción de los canales de distribución. Algunos criterios que el administrador de la red toma en cuenta son: la capacidad de conducción de los canales, la sensibilidad de los cultivos al estrés hídrico (se da prioridad a los cultivos más sensibles), y la cercanía de las parcelas a los canales de distribución.

Para automatizar este procedimiento empírico, se desarrolló un sistema experto (SE), con base en el concepto de ingeniería del conocimiento ("knowledge engineering"), que se resume en la figura 9.
Una vez identificada, conceptualizada, formalizada, implementada y validadas las reglas de inferencia del SE, se codificaron en un programa de cómputo o "shell" denominado Level5 (IBI, 1990) y se acopló al SICODE. El Level5 es un lenguaje para codificar reglas empíricas de inferencia en plataforma windows.

El sistema experto (fig. 10) tiene como principal objetivo establecer las prioridades de riego, en función de las necesidades de los cultivos en periodos de máxima demanda, o después de que ocurre una lluvia más o menos uniforme sobre el distrito de riego, con base en tres criterios: 1. Estrés hídrico de los cultivos, 2. Sensibilidad de los cultivos al estrés hídrico y 3. Distancia de las parcelas a la fuente de suministro más cercana (canal de riego). A continuación se explican estos criterios.

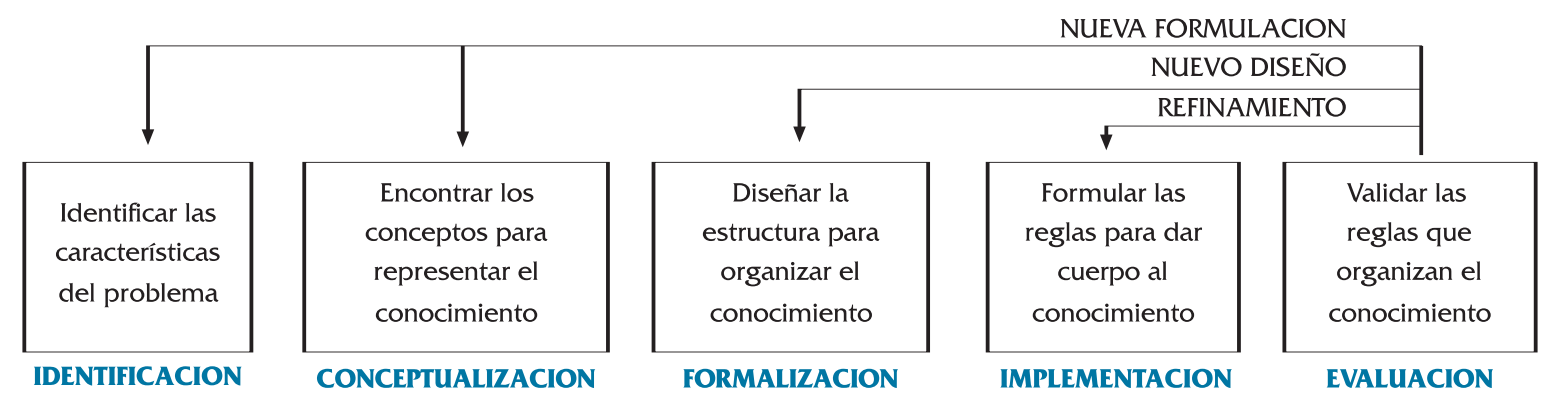

Fig. 9. Ingeniería del conocimiento 
Estrés hídrico. El sistema experto usa los resultados del simulador de balance de agua del SICODE. Con estos datos el SE determina un índice de estrés, definido como la fracción de agua usada por el cultivo, en relación con la cantidad que puede ser extraída del suelo, sin que produzca estrés hídrico a la planta que reduzca su rendimiento.

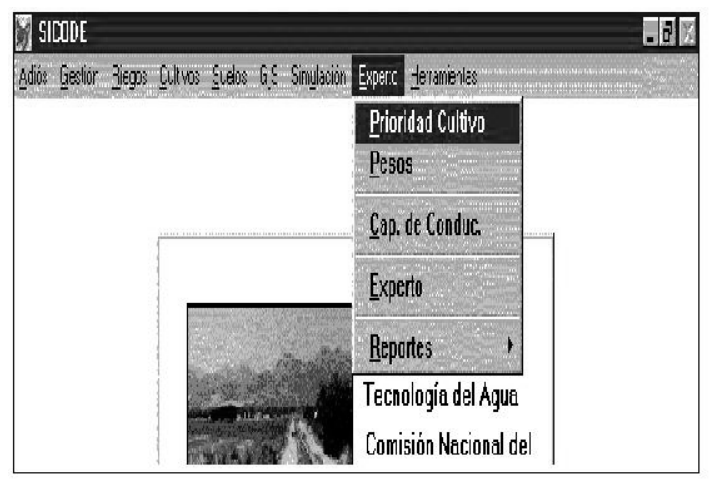

Fig. 10 Menú del sistema experto

Sensibilidad del cultivo. Consiste en una asignación hecha por el usuario, de la sensibilidad relativa al estrés hídrico entre los diferentes cultivos demandantes en un momento dado, en una escala de 1 a 5 . Donde 1 representa la máxima sensibilidad y 5 la mínima sensibilidad.

Distancia de las parcelas a la fuente de suministro (canal de suministro). El SE utiliza como tercer criterio para establecer las prioridades de riego, la cercanía de las parcelas al canal de riego en operación. Se dan prioridad a las parcelas más cercanas a éste $o$ aquellas parcelas inmediatas que acaban de concluir el riego.

\section{CONCLUSIONES}

Se presentó un sistema computarizado denominado SICODE, cuyo objetivo fundamental es apoyar a los usuarios de los distritos de riego en la gestión técnica y administrativa de los mismos.

El SICODE es un sistema que integra de manera modular los siguientes programas: base de datos, sistema de información geográfica, simulación de balance de agua en el suelo, simulador biológico y sistema experto. Incluye también el pronóstico de riego apoyado en estaciones meteorológicas automatizadas. El sistema experto -a diferencia del resto de los programas que componen al SICODE- está basado en reglas empíricas obtenidas de los usuarios en la gestión de sus parcelas. El esquema de inferencia del SE está basado en reglas simples, para lograr una adecuada distribución del agua de riego en períodos de máxima demanda. La base de conocimiento del SE tiene la posibilidad de ser incrementada, incluso con reglas probabilísticas.

Se validó el SICODE en gabinete y en campo en 1995, iniciando las primeras pruebas en el distrito de riego 05, Delicias, Chihuahua, en el norte de México; con esta implementación en Delicias, Chihuahua, se convirtió en pionero en la instalación y uso de estaciones meteorológicas automatizadas para fines de riego en el país. En 1996 inició su divulgación y promoción mediante videos y cursos de capacitación; en ese mismo año se capacitaron a jefes de operación y gerentes de los principales distritos de riego del país. En 1997 inició su transferencia, primero a pequeñas superficies de riego, para luego pasar a áreas cada vez más grandes. Promocionado por los propios usuarios, durante 1997 se transfirió a los distritos de riego 038, en Navojoa, Sonora, y 010, en Culiacán en el estado de Sinaloa.

Debido a la capacidad de gestión (técnica y administrativa), versatilidad, interactividad, características técnicas y de aplicabilidad del SICODE, así como a la solicitud de usuarios y de la Gerencia de Distritos de Riego de la Comisión Nacional del Agua (CNA), actualmente se está usando para gestionar 5000 hectáreas de riego por gravedad en el módulo IV-1 del distrito de riego 010 en Culiacán, Sinaloa.

\section{LISTA DE SIMBOLOS}

Bh Balance de agua en el suelo ( $\mathrm{mm}$ )

Ss ( $\uparrow$ ) Salidas del sistema ( $\mathrm{mm})$

Es $(\downarrow)$ Entradas al sistema (mm)

Pe Precipitación efectiva (mm).

As Contribución del agua subterránea al requerimiento del cultivo (mm).

Aa Agua almacenada antes del riego (mm).

Pt Precipitación total diaria (mm)

Eto Evapotranspiración del cultivo de referencia $\left[\mathrm{mm} \mathrm{d}^{-1}\right]$

$\mathbf{R}_{\mathrm{n}} \quad$ Radiación neta en la superficie de cultivo $\left[\mathrm{MJ} \mathrm{m}^{-2} \mathrm{~d}^{-1}\right]$

G Flujo de calor del suelo $\left[\mathrm{MJ} \mathrm{m}^{-2} \mathrm{~d}^{-1}\right]$

T Temperatura media $\left[{ }^{\circ} \mathrm{C}\right]$

$\mathbf{U}_{2} \quad$ Velocidad del viento a $2 \mathrm{~m}$ de altura $\left[\mathrm{ms}^{-1}\right]$

(ea- ed) Déficit de presión de vapor, [KPa]

$\Delta \quad$ Pendiente de la curva de presión de vapor $\left[\mathrm{KPa}^{\circ} \mathrm{C}^{-1}\right]$ 
$\boldsymbol{\gamma}$ Constante psicrométrica $\left[\mathrm{Kpa}^{\circ} \mathrm{C}^{-1}\right]$

$\boldsymbol{\theta}_{i} \quad$ Humedad residual del suelo (\%)

$\boldsymbol{\theta}_{\mathrm{s}} \quad$ Humedad disponible para el cultivo (\%)

$\boldsymbol{\theta}_{\mathrm{i}+1}^{\mathrm{s}}$ Humedad del suelo en un tiempo posterior $(\%)$

$\boldsymbol{\theta}_{\mathrm{t}} \quad$ Humedad mínima tolerable por el cultivo (\%)

$\boldsymbol{\theta}_{\mathrm{cc}}$ Humedad del suelo en el punto de capacidad de campo (\%)

$\boldsymbol{\theta}_{\text {pmp }}$ Humedad del suelo en el punto de marchitamiento permanente $(\%)$

Dth Descenso tolerable de humedad (\%)

cc Capacidad de campo (\%)

pmp Punto de marchitamiento permanente (\%)

da Densidad aparente del suelo $\left(\mathrm{gr} / \mathrm{cm}^{3}\right)$

\section{REFERENCIAS}

Allen, R.G., Pereira, L.S., RAes, D., and Smith, M. (1998). Crop evapotranspiration: Guidelines for computing crop requirements. Irrigation and Drainage Paper No. 56, FAO, Rome, Italy $300 \mathrm{p}$.

DASTANE, N.G. (1974). Effective rainfall in irrigated agriculture. FAO. Irrigation and Drainage. 25. Rome.

DiCARTU. (1995). Sistema de información geográfica, AU2WIN V.3.0. Manual de usuario, Guadalajara, México.

IBI. (1990). Information Builders, Inc. Level 5 ObjectsOriented Expert System. U.S.A.

IBSNAT (1998). International Benchmark Sites Network for Agrotechnology Transfer. Decision Support System for Agrotechnology Transfer (DSSAT). University of Hawaii. U.S.A.

Mundo Molina M. D., Hernández Leonardo, Bocanegra Antonio, Martínez A. Polioptro. (1995). Capacitación en el uso del sistema computacional para la distribución eficiente del agua en módulos de riego (SICO$D E)$, en: Informe interno anual del Instituto Mexicano de Tecnología del Agua (IMTA), pp. 2.

Mundo Molina M. D. , HernándeZ Leonardo, Bocanegra ANTonio, Martínez A. Polioptro. (1997 a). Transferencia del SICODE a tres módulos de tres distritos de riego del país, Delicias, Chihuahua, Culiacán, Sinaloa y Navojoa, Sonora. Informe final. IMTA. México.

Mundo Molina M. D., Mireles V. Victor., Martínez A. Polioptro. (1977 b). Sistema Computacional para la Distribución Eficiente del Agua en distritos de riego, SICODE, v.2.1. Revista de Ingeniería Hidráulica en México, Vol. XII, Núm. 2, II época, Mayo-agosto, IMTA, México.

Mundo Molina M. D., HeRnándeZ Leonardo, Bocanegra A., Martínez A. Polioptro. (1998). Transferencia del SICODE a tres distritos de riego del país: 05 Delicias, Chihuahua, 038 Culiacán, Sinaloa y 010 Navojoa, Sonora. Informe final. HC-9813. IMTA. México. 